Supplemental Material for

\title{
A Simple Model for Gas-Phase Synthesis of Nickel Nanoparticles
}

\author{
M. R. Kholghy ${ }^{1}$, A. Schumann ${ }^{2}$ \\ Dedicated to Prof. Sotiris E. Pratsinis for his inspiration
}

1. Department of Mechanical and Aerospace Engineering, Carleton University, 1125 Colonel by Drive, Ottawa, K1S 5b6, Canada

2. Department of Mechanical and Process Engineering, ETH Zürich, Sonneggstrasse 3, Zürich CH-8092, Switzerland

*Corresponding Author: reza.kholghy@carleton.ca 


\section{Neck growth rate vs normalized neck radius at different temperatures}

Here the neck growth rate of a nickel dimer is calculated using the relation suggested by Coblenz $^{49}$ :

$$
\frac{d\left(\frac{x}{r}\right)}{d t}=\frac{(25 / 6) D_{s} \delta_{s} \gamma_{s} v_{a}}{R T r^{4}}\left(\frac{x}{r}\right)^{-5}
$$

which results in the following relation for the characteristic sintering time of nickel with surface diffusion:

$$
\tau_{S_{-} S_{-} \text {Coblenz }}=2.31 \cdot 10^{23} \cdot T \cdot d_{p}^{4} \cdot \exp \left(\frac{60000}{R T}\right)
$$

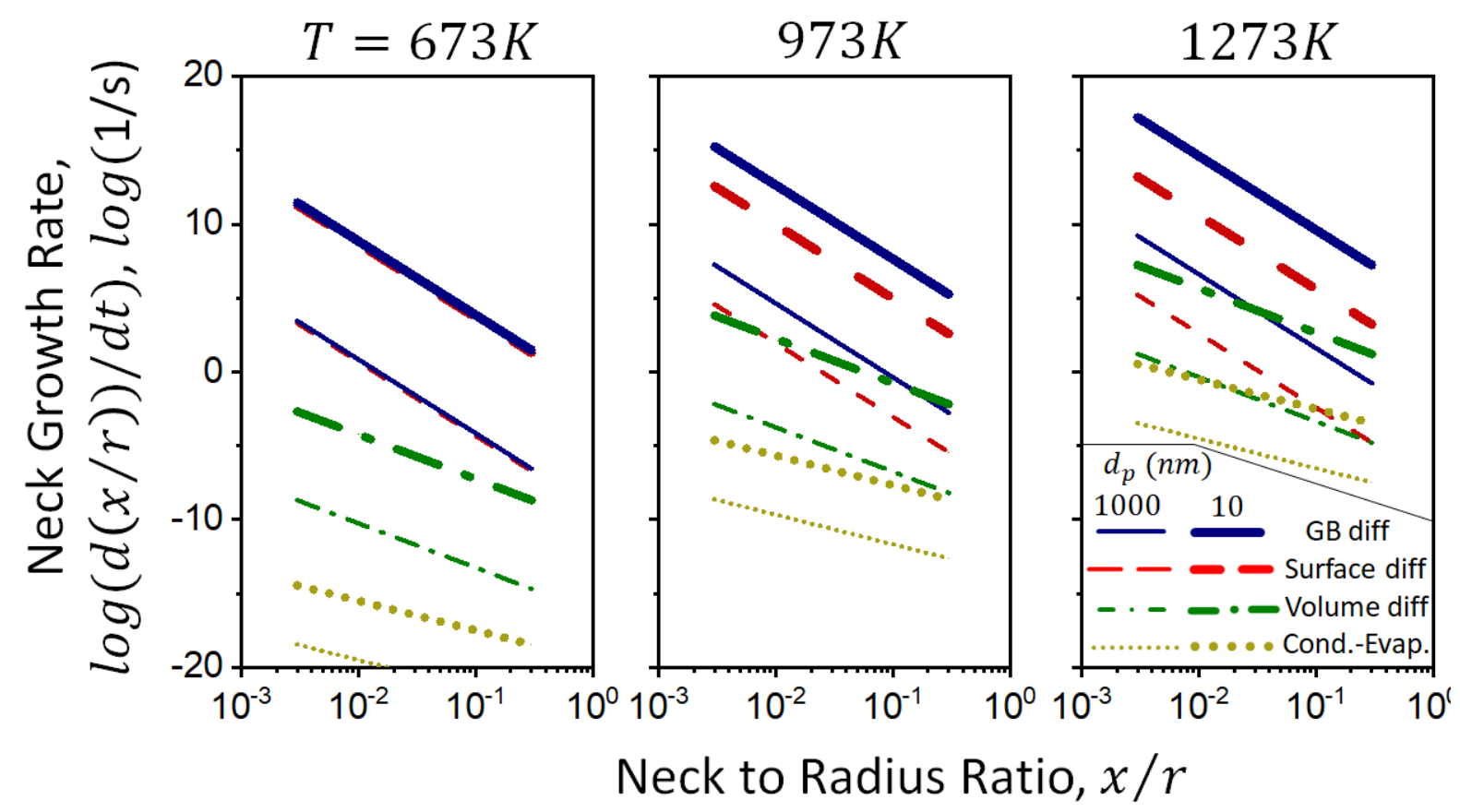

Figure S1. Neck growth rate, $d(x / r) / d t$, by various sintering mechanisms as a function of neck to radius ratio, $x / r$, at $T=673,973$, and $1273 K$, for two $N i$ spheres with initial diameter of, $d_{p}=10$ (thick lines) and $1000 \mathrm{~nm}$ (thin lines). Surface diffusion here (dashed lines, Eq. S1) is calculated with the formula of Coblenz ${ }^{49}$ and is lower than grain boundary diffusion (solid lines, Eq. 19) at all and $x / r$. Here, grain boundary diffusion is the dominant sintering mechanism followed by surface diffusion (dashed lines, Eq. S1), volume diffusion (dash dotted lines, Eq. 20) and evaporation-condensation (dotted lines, Eq. 21).

\section{Derivation of characteristic sintering times from neck growth rates}

Rearranging Eq. 18 yields:

$\left(\frac{x}{r}\right)^{6} d\left(\frac{x}{r}\right)=\frac{8 D_{s} \delta_{s} \gamma_{s} v_{a}}{R T r^{4}} d t \stackrel{d r \ll d\left(\frac{x}{r}\right)}{\longrightarrow} \int_{0}^{0.83}\left(\frac{x}{r}\right)^{6} d\left(\frac{x}{r}\right)=\int_{0}^{\tau_{s}} \frac{8 D_{s} \delta_{s} \gamma_{s} v_{a}}{R T r^{4}} d t$ 


$$
\begin{aligned}
& \rightarrow \frac{\left(\frac{x}{r}\right)^{7}}{7}=\frac{8 D_{s} \delta_{s} \gamma_{s} v_{a}}{R T r^{4}} \tau_{s} \rightarrow \tau_{s}=\frac{\frac{(0.83)^{7}}{7} R T r^{4}}{8 D_{s} \delta_{s} \gamma_{s} v_{a}}=\frac{(0.83)^{7} R T d_{p}^{4}}{7 \cdot 8 \cdot 16 \cdot D_{s} \delta_{s} \gamma_{s} v_{a}} \\
& \rightarrow \frac{(0.83)^{7} \cdot 8.314 \cdot T \cdot d_{p}^{4}}{7 \cdot 8 \cdot 16 \cdot 9 \cdot 10^{-12} \cdot \exp (-60000 / R T) \cdot 2.48 \cdot 10^{-10} \cdot 2 \cdot \frac{0.0587}{8908}} \\
& \rightarrow \tau_{S_{-} S D}=8.56 \cdot 10^{22} \cdot T \cdot d_{p}^{4} \cdot \exp (60000 / R T)
\end{aligned}
$$

Similarly, by rearranging Eq. 19 we obtain:

$$
\begin{aligned}
& \left(\frac{x}{r}\right)^{5} d\left(\frac{x}{r}\right)=\frac{8 D_{g b} \delta_{g b} \gamma_{s} v_{a}}{R T r^{4}} d t \stackrel{d r \ll d\left(\frac{x}{r}\right)}{\longrightarrow} \int_{0}^{0.83}\left(\frac{x}{r}\right)^{5} d\left(\frac{x}{r}\right)=\int_{0}^{\tau_{s}} \frac{8 D_{g b} \delta_{g b} \gamma_{s} v_{a}}{R T r^{4}} d t \\
& \rightarrow \frac{\left(\frac{x}{r}\right)^{6}}{6}=\frac{8 D_{g b} \delta_{g b} \gamma_{s} v_{a}}{R T r^{4}} \tau_{s} \rightarrow \tau_{s}=\frac{\frac{(0.83)^{6}}{6} R T r^{4}}{8 D_{g b} \delta_{g b} \gamma_{s} v_{a}}=\frac{(0.83)^{6} R T d_{p}^{4}}{6 \cdot 8 \cdot 16 \cdot D_{g b} \delta_{g b} \gamma_{s} v_{a}} \\
& \rightarrow \frac{(0.83)^{6} \cdot 8.314 \cdot T \cdot d_{p}^{4}}{6 \cdot 8 \cdot 16 \cdot 4 \cdot 10^{-4} \cdot \exp (-165000 / R T) \cdot 6 \cdot 10^{-10} \cdot 2 \cdot \frac{0.0587}{8908}} \\
& \rightarrow \tau_{s_{-} g b}=1.12 \cdot 10^{15} \cdot T \cdot d_{p}^{4} \cdot \exp (165000 / R T)
\end{aligned}
$$

\section{Calculating agglomerate surface area from its measured $d_{m}$ and $d_{p}$}

Equivalent agglomerate volume, $v_{e q}$, can be written as a function of $d_{m}$ and $d_{p}$ :

$$
v_{e q}=n_{p} \frac{\pi}{6} d_{p}^{3}(@ t=0)=k_{m}\left(\frac{d_{m}}{d_{p}}\right)^{D_{f m}} \frac{\pi}{6} d_{p}^{3}(@ t=0)
$$

where $k_{m}$ and $D_{f m}$ are mass-mobility prefactor and exponent, respectively. Then, the area of a sphere with the same volume as the agglomerate, $a_{e q}$, is:

$$
a_{e q}=\pi\left(n_{p} d_{p}^{3}\right)^{\frac{2}{3}}(@ t=0)=\pi\left(k_{m}\left(\frac{d_{m}}{d_{p}}\right)^{D_{f m}} d_{p}^{3}\right)^{\frac{2}{3}}(@ t=0)
$$

During sintering, agglomerate volume does not change. However, its area decreases and the surface equivalent primary particle diameter changes according to:

$$
d_{p}=6 \frac{v_{e q}}{a}
$$

and the mobility diameter evolves according to:

$$
d_{m}=d_{p}\left(\left(\frac{a_{e q}}{\pi}\right)^{\frac{3}{2}} \frac{1}{k_{m}} \frac{1}{d_{p}^{3}}\right)^{\frac{1}{D_{f m}}}
$$


Thus, with the knowledge of initial agglomerate $d_{m}(0)$ and $d_{p}(0), v_{e q}$ and $a_{e q}$ can be calculated and with Eq. 17 evolution of agglomerate surface area during sintering, is calculated which will be used to calcaute new $d_{p}$ and $d_{m}$ from Eq. S5 and S6, repectively in Fig. 2.

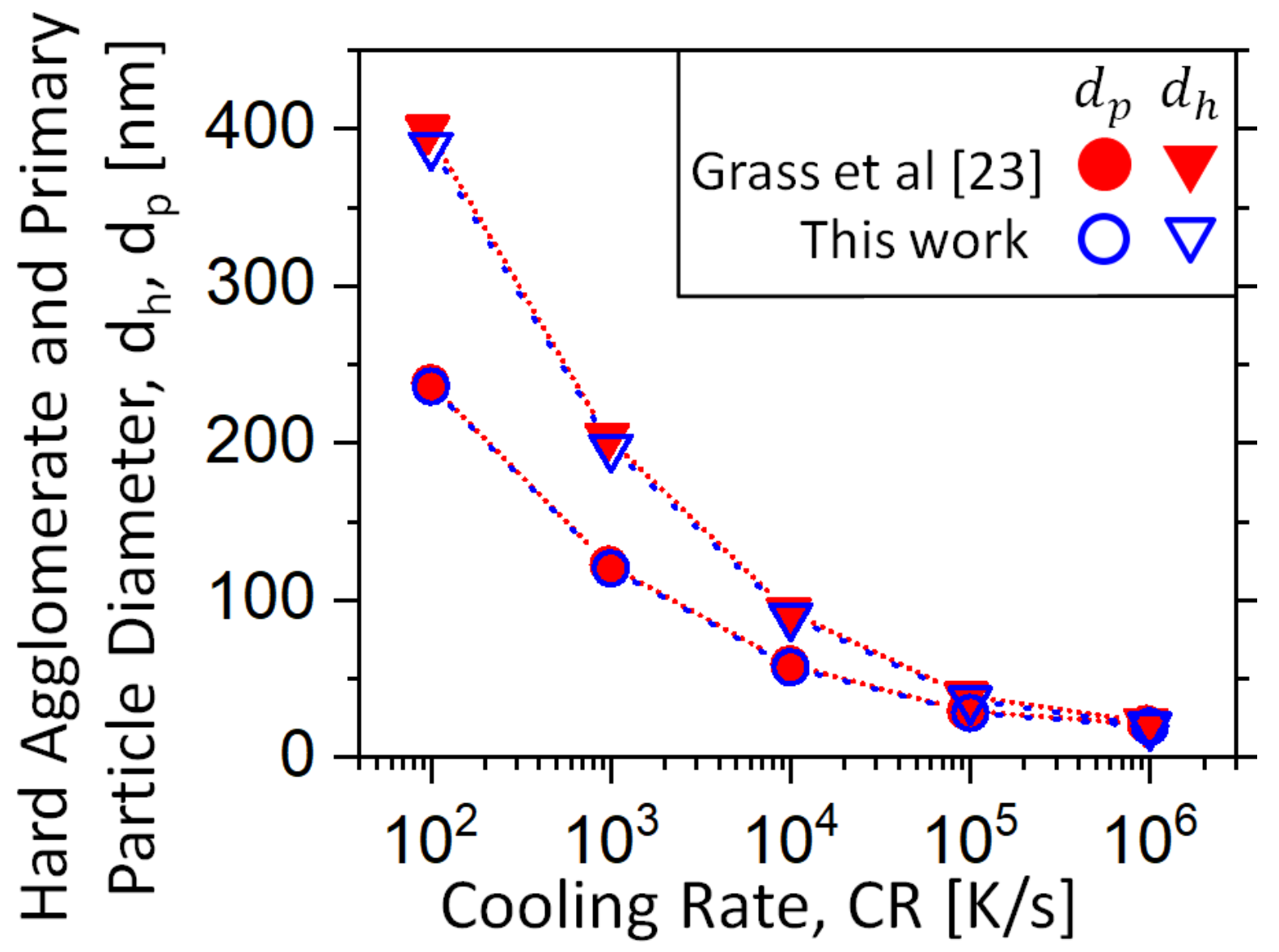

Figure $\mathrm{S} 2 . \mathrm{TiO}_{2}$ primary particle, $d_{p}$, and hard agglomerate, $d_{h}$, diameters at $T_{\max }=$ $2200 \mathrm{~K}$ (reached at $2 \mathrm{~ms}$ ), $\varphi=0.01$ and different cooling ratios simulated by Grass et al. ${ }^{23}$ ( their Figure 5) (filled symbols) and the MPBM of this work (empty symbols). 


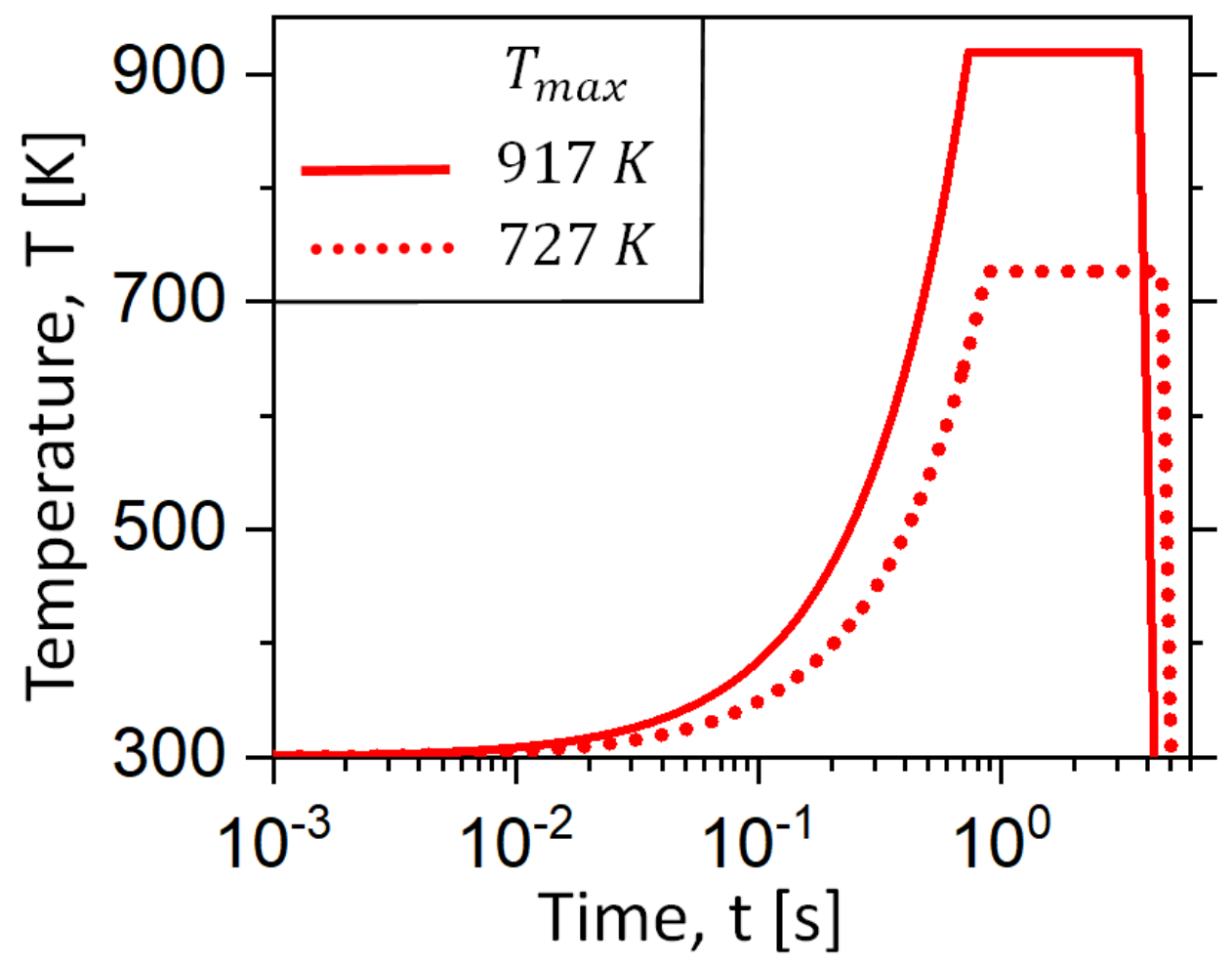

Figure S3. Reactor temperature profile vs time ${ }^{16}$. 
Average primary particle diaemters areobtained from TEM images provided for experiment 020501 in the $\mathrm{PhD}$ thesis of Eric B. Wasmund ${ }^{17}$, Chapter, 4, pages, 119 to 112. Images and selected particles are shown in the figures below.

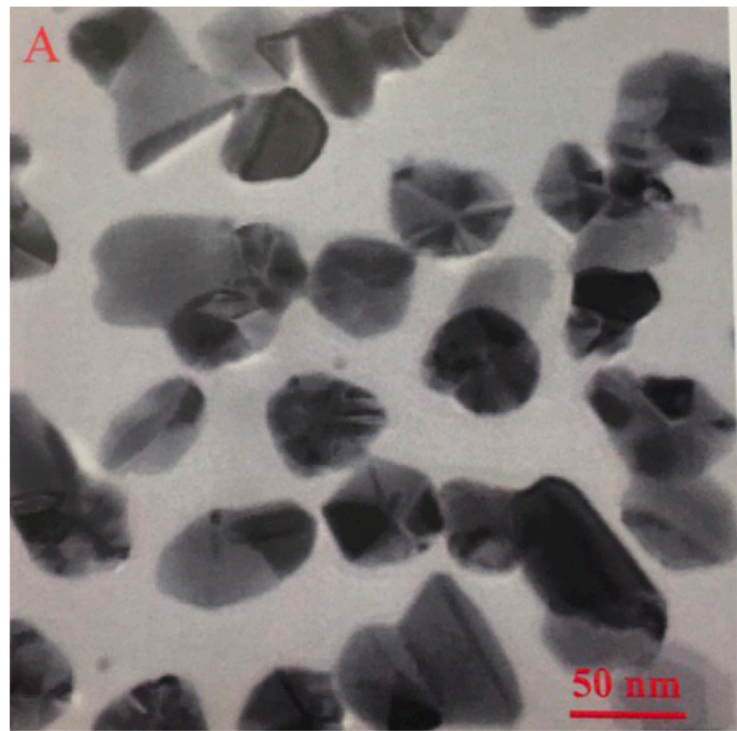

(a) $5 \mathrm{~cm}$ above inlet.

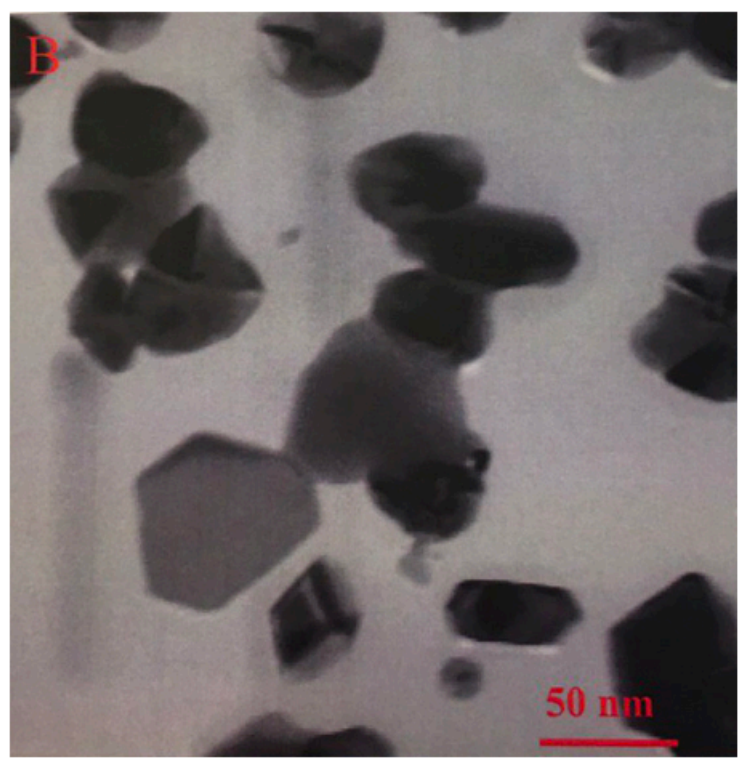

(c) $5 \mathrm{~cm}$ above inlet.

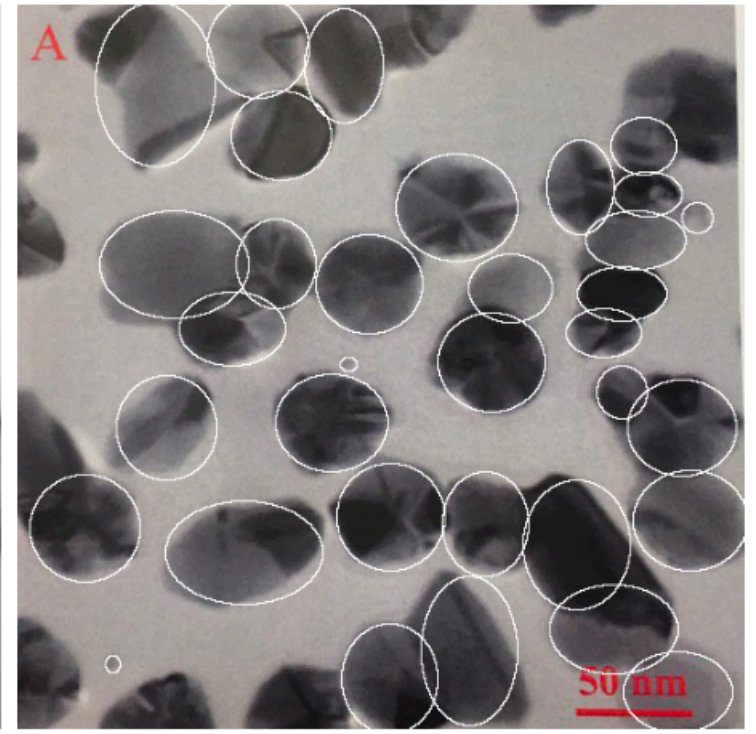

(b) Circled particles are considered primary particles.

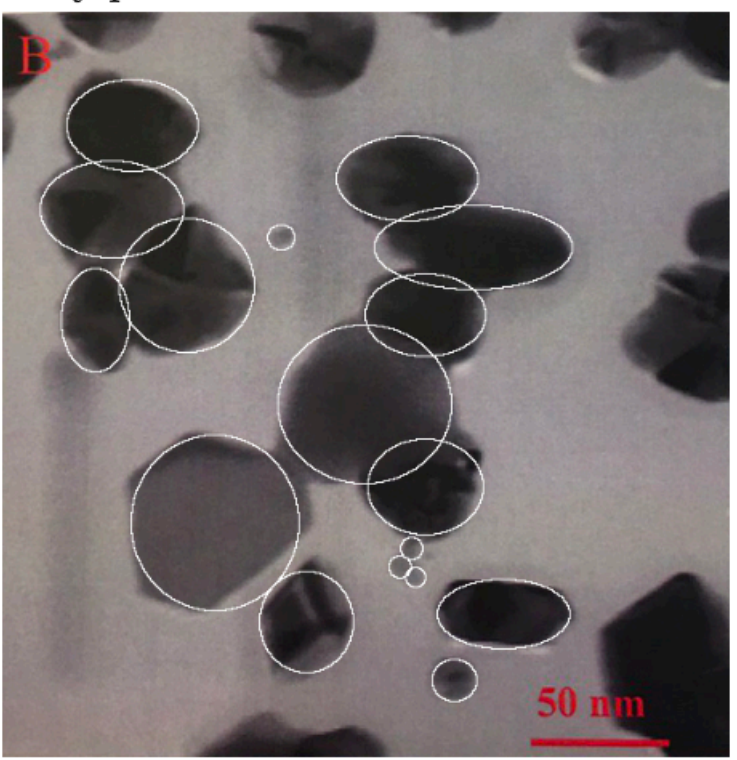

(d) Circled particles are considered primary particles.

Fig. S4. The geometric mean primary particle diameter is $\mathrm{dp}$, mean $=32 \mathrm{~nm}$ and the geometric standard deviation is $\sigma \mathrm{g}=1.83$. 


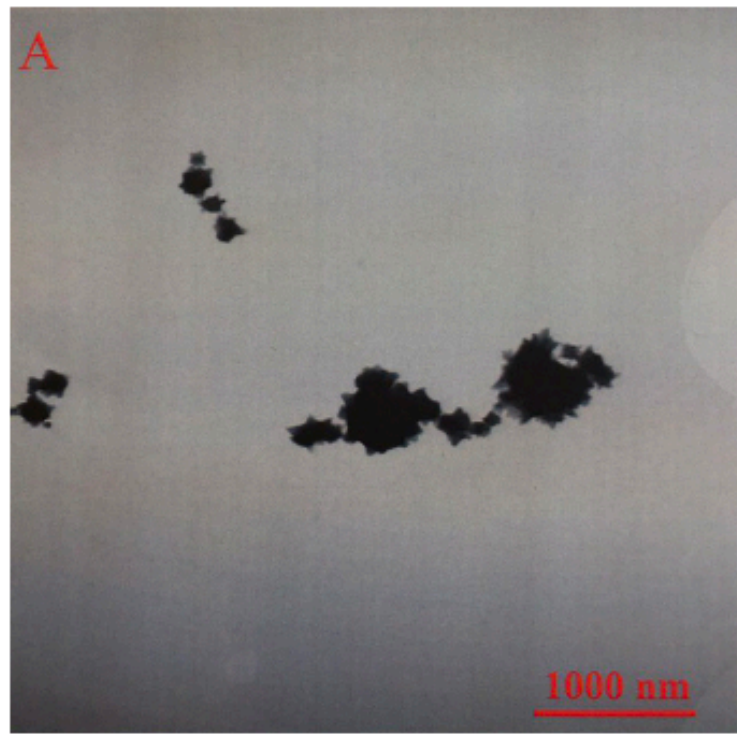

(a) $15 \mathrm{~cm}$ above inlet.

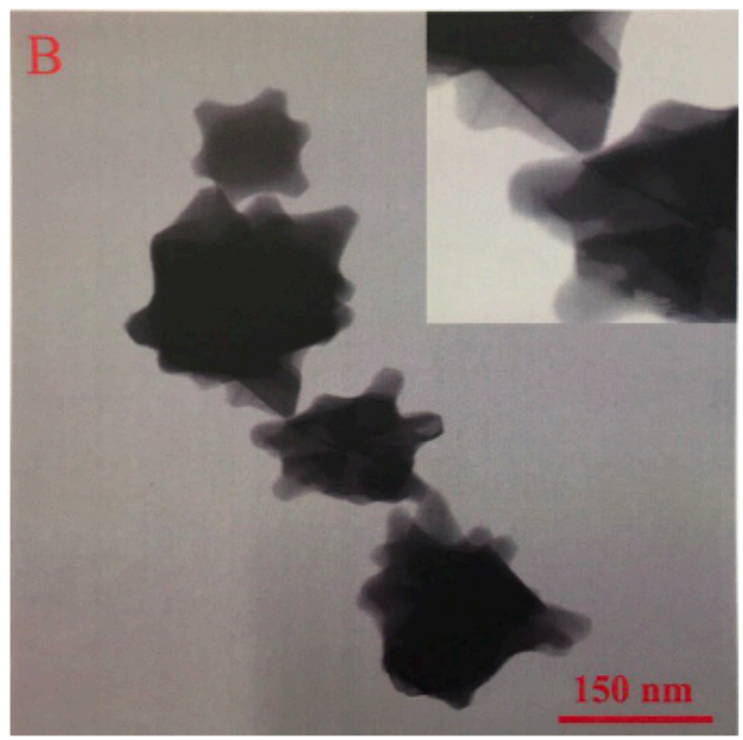

(c) $15 \mathrm{~cm}$ above inlet.

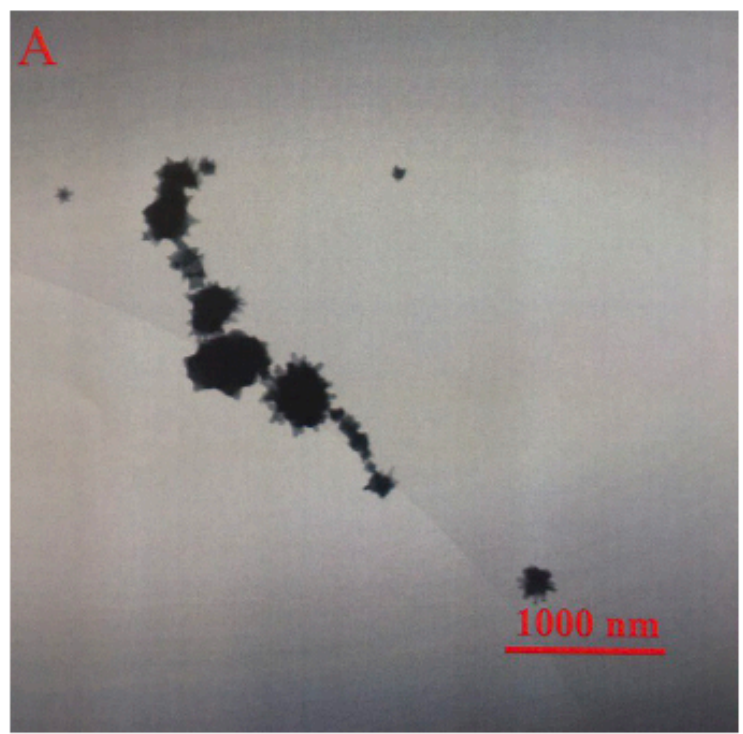

(e) $15 \mathrm{~cm}$ above inlet.

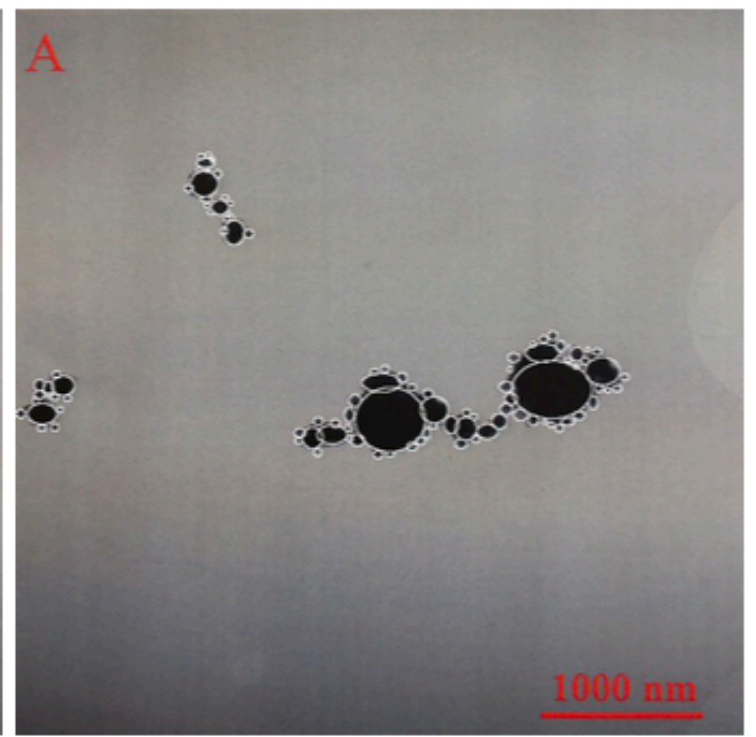

(b) Circled particles are considered primary particles.

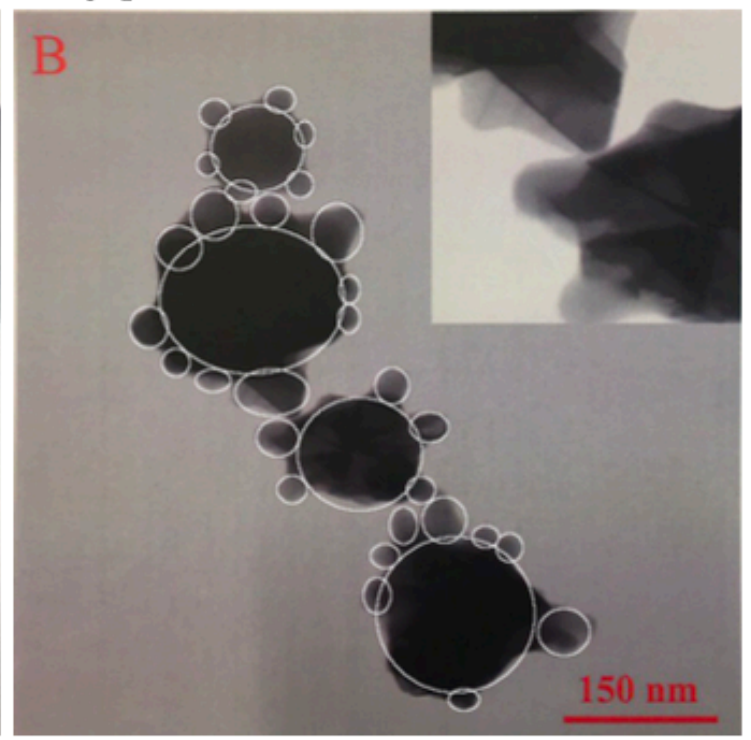

(d) Circled particles are considered primary particles.

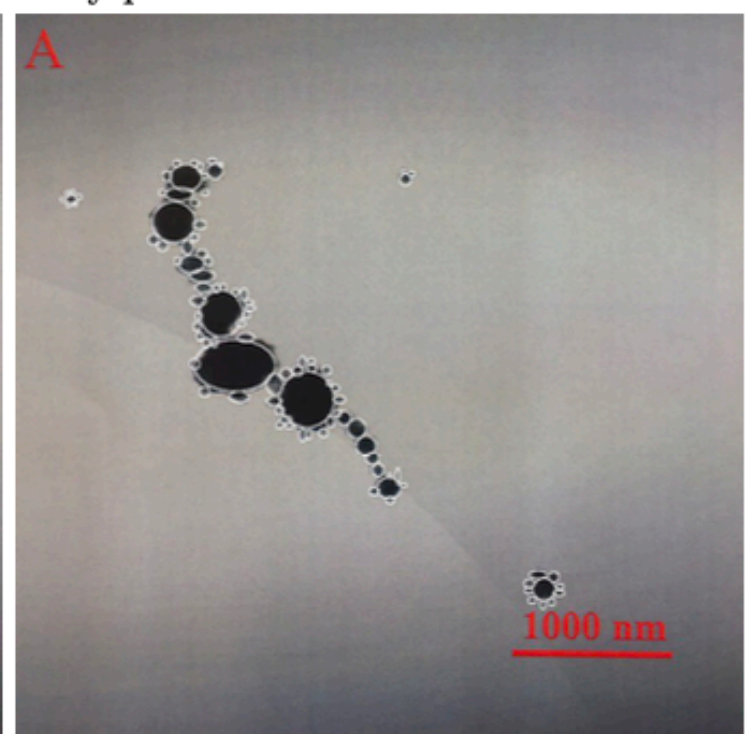

(f) Circled particles are considered primary particles. 


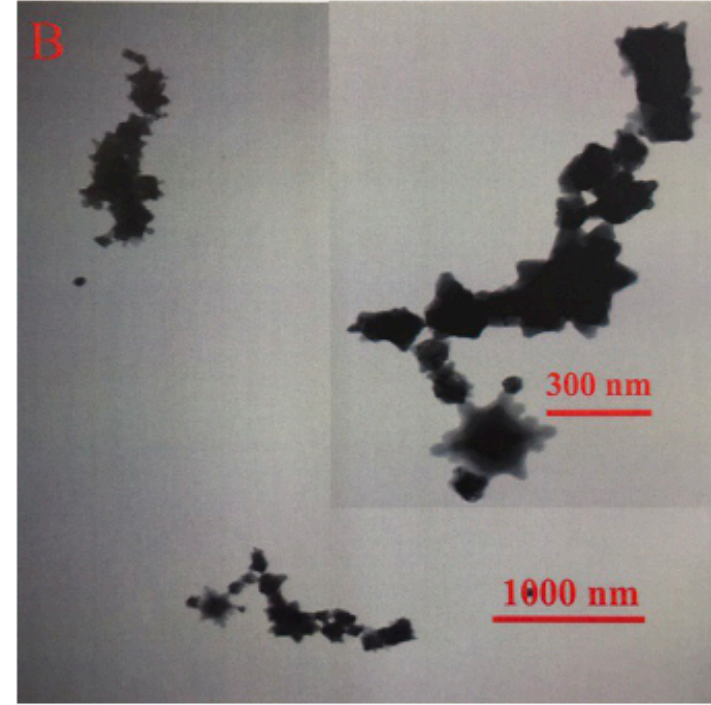

(g) $15 \mathrm{~cm}$ above inlet.

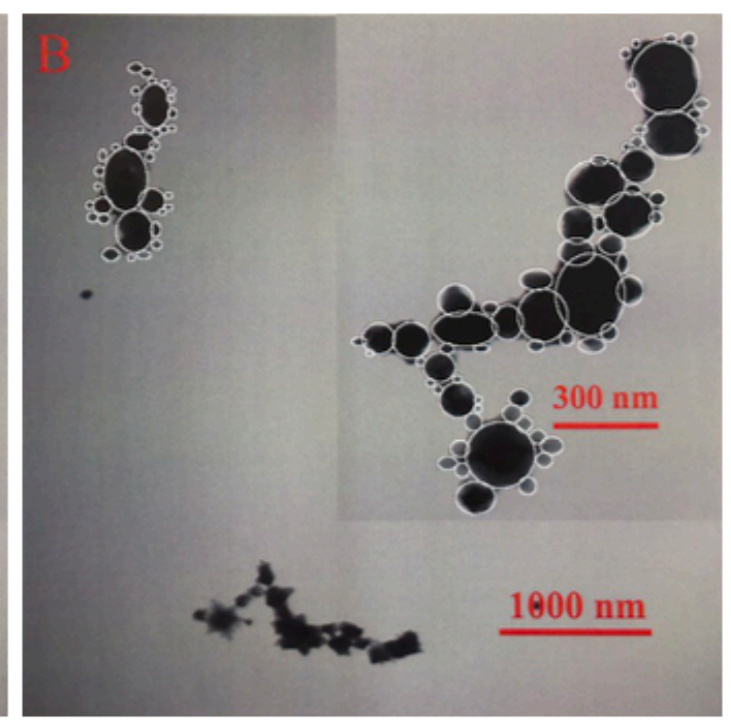

(h) Circled particles are considered primary particles.

Fig. S5. The geometric mean primary particle diameter is $\mathrm{dp}$, mean $=55 \mathrm{~nm}$ and the geometric standard deviation is $\sigma \mathrm{g}=1.79$. 


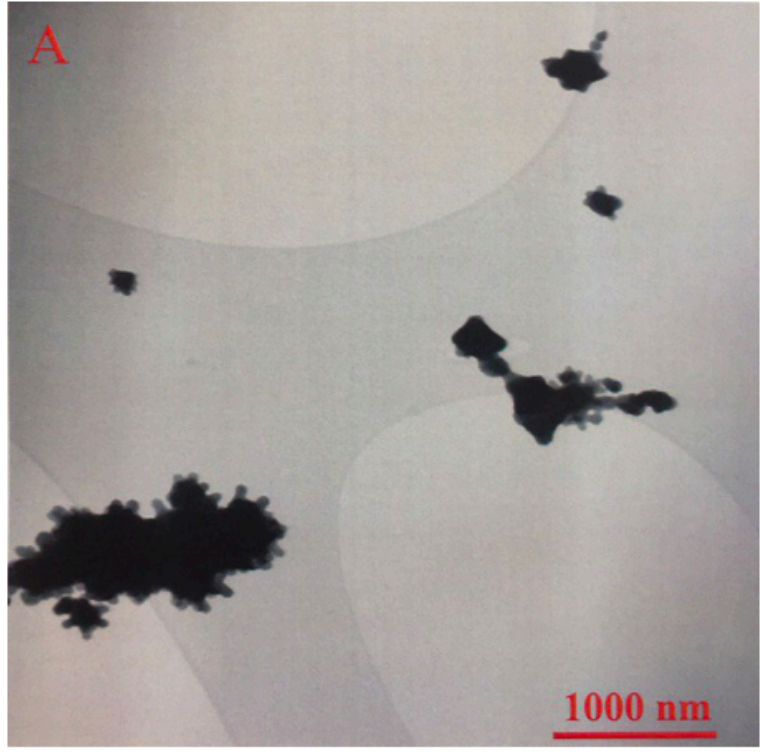

(a) $25 \mathrm{~cm}$ above inlet.

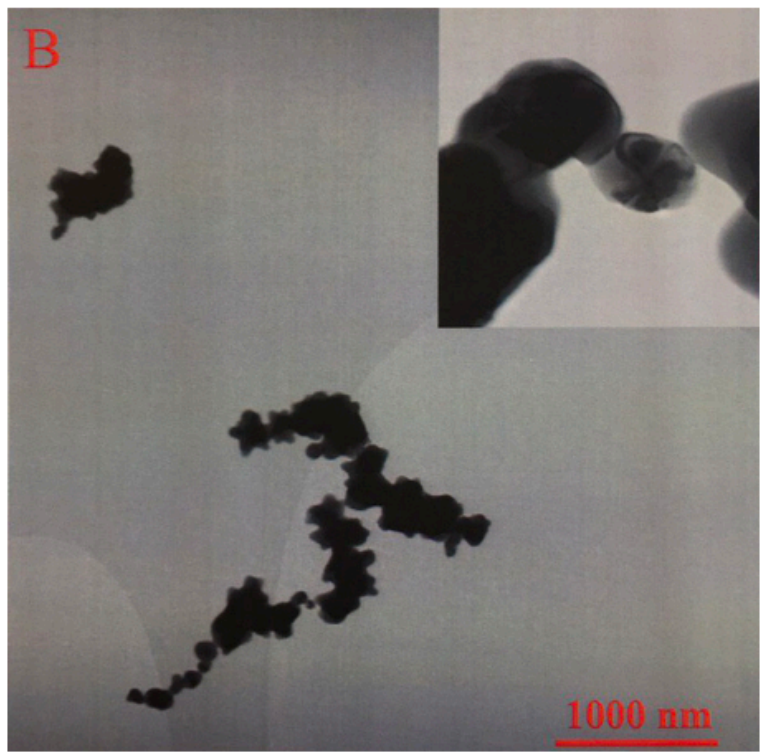

(c) $25 \mathrm{~cm}$ above inlet.

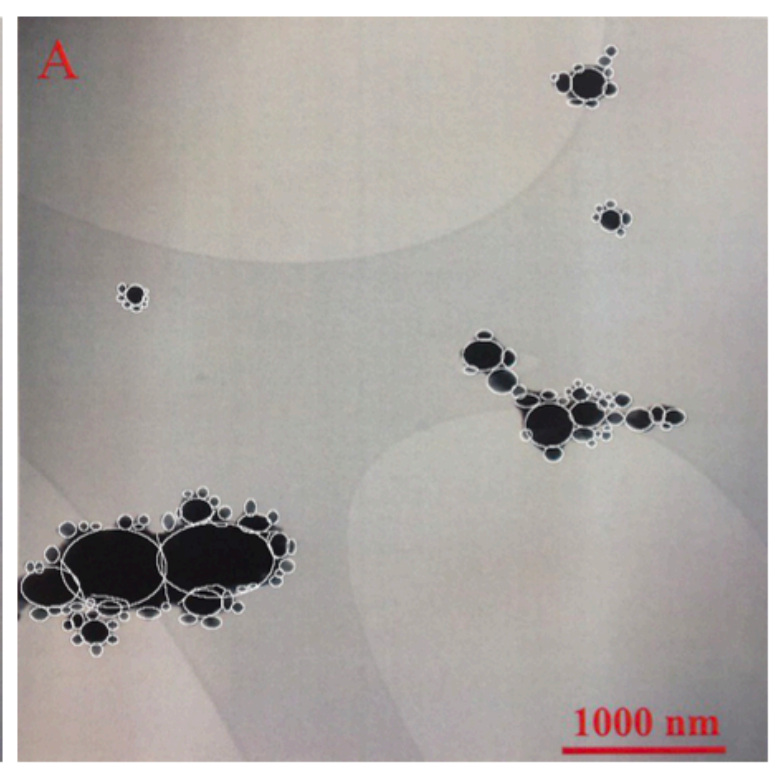

(b) Circled particles are considered primary particles.

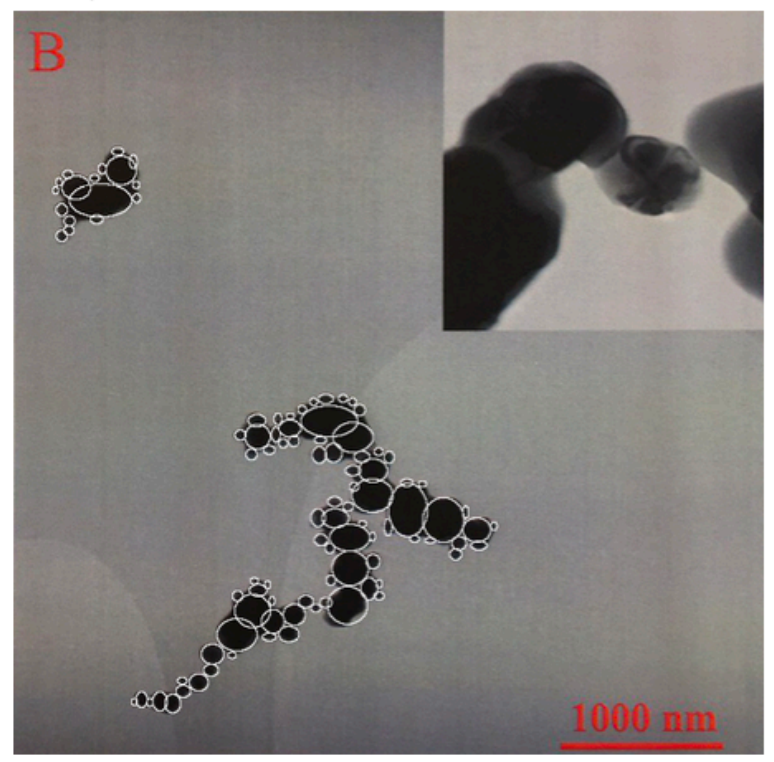

(d) Circled particles are considered primary particles.

Fig. S6. The geometric mean primary particle diameter is $d p, m e a n=78 \mathrm{~nm}$ and the geometric standard deviation is $\sigma g=1.70$. 